Revue d'histoire de l'Amérique française

QAS REVUE D.HISTOIRE DE L'AMÉRIQUE FRANÇAISE

\title{
Nicolas Perrot a-t-il tenté d'empoisonner Cavelier de la Salle ?
}

\section{J.-Alfred Perrault}

Volume 1, numéro 1, juin 1947

URI : https://id.erudit.org/iderudit/801345ar

DOI : https://doi.org/10.7202/801345ar

Aller au sommaire du numéro

Éditeur(s)

Institut d'histoire de l'Amérique française

ISSN

0035-2357 (imprimé)

1492-1383 (numérique)

Découvrir la revue

Citer cet article

Perrault, J.-A. (1947). Nicolas Perrot a-t-il tenté d'empoisonner Cavelier de la Salle ? Revue d'histoire de l'Amérique française, 1(1), 49-53.

https://doi.org/10.7202/801345ar d'utilisation que vous pouvez consulter en ligne.

https://apropos.erudit.org/fr/usagers/politique-dutilisation/ 


\section{NICOLAS PERROT A-T-IL TENTÉ D'EMPOISONNER CAVELIER DE LA SALLE ?}

Chacun connait Pierre Margry, historien français du dix-neuvième siècle, spécialiste de l'histoire maritime et coloniale de la France, directeur des archives de la marine et des colonies à Paris. En 1880 Margry accuse Nicolas Perrot, grand explorateur et découvreur des pays d'en haut, Illinois et North Dakota, d'une tentative de meurtre par empoisonnement contre le Sieur Cavelier de La Salle. Cette accusation est portée dans l'ouvrage de l'historien, qui a pour titre: Découvertes et Établissements des Français dans l'ouest et dans le sud de l'Amérique septentrionale (1614-1754) - Mémoires et documents originaux, cinquième partie: Première formation d'une Chaine de postes entre le fleuve Saint-Laurent et le Golfe du Mexique (1683-1724), Introduction, p. XXXIV. Margry y écrit en toutes lettres: "On avait vu même Nicolas Perrot, qui avait tenté d'empoisonner l'illustre Découvreur, devenir un des chefs de poste de l'Ouest.) Et Margry nous renvoie au premier volume de: “Découvertes et Établissements des Français... p. 370. En réalité le renseignement est plutôt aux pages 389-90; et il est de l'( Histoire de M. de La Salle », intercalée dans un " Récit de l'abbé de Gallinée ".

Rétablissons les faits. Nous sommes en 1676 ou 1677. La Salle se trouve au Fort Frontenac dont il a reçu le commandement. Les Jésuites - toujours d'après l'auteur de l'“ Histoire de M. de La Salle ") - et pour des motifs que cet auteur, ni nous-mêmes n'apercevons bien, se seraient appliqués à semer des bruits de guerre, en vue d'indisposer les Iroquois contre La Salle et les Français. Frontenac, escorté seulement d'une cinquantaine d'hommes, se serait rendu au poste de La Salle pour calmer l'inquiêtude des cantons. C'est pendant ce séjour du gouverneur au Fort Frontenac que la tentative d'empoisonnement aurait eu lieu. Et voici comment l'" Histoire de M. de La Salle " la raconte: "Quoy qu'il en soit, M. de La Salle se sentit 
quelque temps après empoisonné d'une salade dans laquelle on avoit meslé beaucoup de ciguë, qui est poison en ce pays-là, et du verd de gris. Il en fut malade à l'extrémité, vomissant presque continuellement quarante ou cinquante jours après, et il n'eschappa que par la force extrême de sa constitution. Celuy qui lui donna le poison fut un nommé Nicolas Perrot, autrement Joly-Cœur, l'un de ses domestiques. Il n'est pas probable que ce malheureux ait fait une si meschante action de son propre mouvement, parce que M. de La Salle est fort aimé de ses domestiques, qui font de grands profits par les occasions qu'il leur donne de trafiquer, comme j'ay appris d'une personne désintéressée depuis qu'il est party. »)

Francis Parkman, l'historien américain, a malheureusement fait un sort à l'accusation de Margry, dont il se défie trop peu. Dans son "La Salle and the Discovery of the Great West " ${ }^{1}$, il commente comme suit l'histoire fantaisiste: "Ceci nous montre le caractère die Perrot dans une lumière nouvelle; car il ne semble pas qu'il s'agisse en l'espèce d'un autre personnage que le fameux voyageur. Je n'ai trouvé nulle part ailleurs qu'on fît mention du synonyme de Joly-cœur. L'empoisonnement était alors le crime à la mode et des personnages du plus haut rang en avaient été maintes fois accusés $).{ }^{2}$.

Malheureusement pour Margry et Parkman, Nicolas Perrot, le voyageur, n'était pas alors et ne pouvait être un domestique. Nicolas Perrot, à la date de cette affaire d'empoisonnement, comptait parmi les hommes les plus prospères du Canada. Il voyageait beaucoup, avait obtenu pour lui-même des congés et traitait pour son propre compte. En 1671, comme en fait foi le document de la prise de possession "dudit lieu Sainte-Marie-du-Saut ), il signe comme " interprète pour Sa Majesté en cette partie ». Nulle part je n'ai trouvé qu'il se soit engagé à qui que ce soit, si ce n'est aux Jésuites et aux MM. de

1. Parkman (Francis), La Salle and the Discovery of the Great West-France and England in North America, Part Third, Boston, Little, Brown, and Company, 1942 , p. 116.

2. "This puts the character of Perrot in a new light; for it is not likely that any other can be meant than the famous voyageur. I have found no mention elsewhere of the synonyme of Jolycœur. Poisoning was the current crime of the day, and persons of the highest rank had repeatedly been charged with it." - Parkman (Francis), La Salle and the Discovery of the Great West - France and England in North America - Part third, Boston, Little, Brown, and Company, 1942, p. 116. 
Saint-Sulpice, et ceci, dès le début de sa carrière de coureur des bois, $1660-1668$.

Nicolas Perrot est alors marié à Magdelaine Raclot qui lui a apporté du bien. Il a trois ou quatre enfants, une bonne terre en culture à Rivière St-Michel, Bécancourt, avec maison, bâtiments, bestiaux. Il fait plusieurs transactions comme le font voir les documents laissés dans différents greffes de notaires des Trois-Rivières, de Montréal, de Québec. Il a lui-même des domestiques, Jean Guittard, Jean Boutillé. Ce dernier s'est donné à Nicolas Perrot, le 24 juin 1675, par acte passé devant Mtre Guillaume De La Rue, notaire à Champlain. Et voici l'acte: "Le dit Jean boutillé, de son bon gré, " pure et franche vollonté, sans aucune force ni contrainte, a recognu " par les présentes sestre donné pour travailler pendant savye du(" rantte au S. Nicollas perot pour lequel ledict boutillé s'est obligé de " commancer à entrer au servisse du dict $\mathrm{S}$. Perot au premier jour de ( juillet prochain venant et continuera toute savye durant sans aucune “ intervalle et réservation de tout le tant... tant qu'il plaira à Dieu le " créateur du ciel et de la terre le lesé vivre sur terre dont il s'oblige " de bien et d'heuman servir le dict S. Perot en tout ce qui luy sera "que mandé de faire ", etc.

Nicolas Perrot n'a pas qu'un seul " engagé ». Il en a plusieurs pour faire la traite dans "les pays lointains". Anthoine Adhémar, notaire, greffe de Montréal, a fait, à cette époque, de nombreux contrats d'engagements à Nicolas Perrot. Il est donc impossible que Nicolas Perrot se soit engagé à La Salle et comme domestique. Cependant, il se peut que Cavelier de La Salle ait eu un serviteur du nom de Nicola Perrot. Car, en 1690, le 24 avril, devant Mtre Genaple, notaire à Québec, un certain Nicola Perrot, ancien domestique des Jésuites, au collège, s'engage de nouveau pour trois ans, comme domestique. Ce Nicola Perrot déclare ne savoir signer; ce qui n'est pas le cas de Nicolas Perrot, le découvreur, qui est, comme l'on sait, de bonne éducation, instruit et qui toujours signe, quand requis de le faire. La même année, devant le même notaire, trois mois plus tôt, le 24 février 1690, Nicolas Perrot signe un acte de vente par Charles Damour de Louvier à lui-même, Nicolas Perrot, de certains droits et part d'une société que Charles de Louvier a conclu avec Nicolas Perrot pour la traite des fourrures. 
Il y a donc, à la même époque, deux Nicolas Perrot au pays. Parkman n'avait pas rencontré, dans ses recherches, le second personnage, c'est-à-dire le domestique, et Margry encore moins. Du reste, sans y apporter peut-être toutes les preuves qu'il y aurait fallu, M. Henri Lorin, dans: Le Comte de Frontenac-Etude sur le Canada français a la fin du XVIIe siècle, (Paris, Armand Colin, 1895), avait déjà apporté une dénégation à l'encontre de la fantaisie MargryParkman. On lit, en effet, aux pages 202-203 de l'ouvrage:

" Pendant son séjour au fort en 1676 ou 1677, La Salle fut victime d'une tentative d'empoisonnement; on lui avait, paraît-il, versé de la ciguë et du vert-de-gris dans une salade; sa santé, fort compromise pendant quelques jours, se rétablit cependant, et le mal ne laissa point de traces. Dans son entourage, quelques-uns avaient accusé les Jésuites; mais La Salle reconnaît loyalement que les Pères n'y sont pour rien. Aussi bien le document janséniste de Margry est-il le seul à mentionner cette tentative, d'après des fragments de lettres de La Salle dont nous ne pouvons vérifier l'authenticité. Mais il contient évidemment une erreur lorsqu'il dénonce comme auteur du crime " un nommé Nicolas Perrot, autrement Jolycœur ). Très probablement La Salle fut victime d'une vengeance particulière; il n'était pas aimé de ses domestiques, de ses compagnons de voyage, dont il exigeait ce qu'il donnait lui-même de courage et d'endurance, ne comprenant pas qu'il leur manquait la force de sa foi, qui n'animait que lui. Un domestique, un soldat, peut-être bien le Jolycœur dont parle la revue de 1677 put lui garder rancune d'une réprimande, et tenter sans succès un crime que d'autres, dans des conditions certainement analogues, accompliront plus tard; mais ce Jolycœur n'a rien de commun avec Nicolas Perrot, le célèbre coureur de bois. Nicolas Perrot n'a jamais été le compagnon de La Salle, dont il ne prononce qu'une fois le nom, comme celui d'un étranger rencontré par hasard en 1670, au cours d'une expédition de chasse. En 1676 et 1677, il voyageait sans doute comme auparavant, parmi les populations de l'ouest du lac Michigan; on ne voit d'ailleurs aucune raison qui pourrait expliquer, de sa part, un acte d'hostilité contre La Salle; et Parkman, au lieu d'accepter la version du document de Margry, en s'étonnant seulement de ce surnom de Jolycœur que nulle part ailleurs Perrot n'a porté jamais, eût mieux fait de conclure que l'auteur de ce pamphlet, peu familier avec les choses canadiennes, a confondu deux personnages en un seul. 
La discussion n'aurait point d'importance, si le résultat n'en était de placer dans l'entourage immédiat de La Salle le criminel qui voulut l'empoisonner. ")

Je pense que mon ancêtre est ainsi lavé pour toujours de la sotte accusation. En fin de ce petit article, me permettra-t-on d'ajouter qu'il y aurait un magnifique, et des plus intéressant travail à faire sur Nicolas Perrot? On trouve à tout moment des documents qui le concernent, soit aux Archives de Montréal, de Québec ou des TroisRivières. Pour ma part, je possède un bon nombre de copies de ces documents et, encore plus, une liste d'autres pièces à copier et qui semblent n'avoir jamais été publiées. En ouvrant un de ces documents, j'y ai même trouvé le sable dont on se servait pour assécher l'encre.

Il me ferait plaisir de prêter toute ma documentation à qui voudrait entreprendre un tel travail qui serait vraiment inédit.

J.-Alfred Perrault,

de la Société généalogique canadienne-française, (Outremont, Québec). 